\section{P176 HIV ACQUISITION AND ANTIRETROVIRAL THERAPY INITIATION IN A YOUTH COHORT IN SOWETO AND DURBAN, SOUTH AFRICA}

${ }^{1}$ Stefanie Hornschuh, ${ }^{1}$ Fatima Laher, ${ }^{1}$ Kennedy Otwombe, ${ }^{2}$ Patricia Smith, ${ }^{3}$ Mags Beksinska, ${ }^{4}$ Glenda Gray, ${ }^{2}$ Mark Brockman, ${ }^{3}$ Jenni Smit, ${ }^{2}$ Angela Kaida*, ${ }^{1}$ Janan Dietrich. ${ }^{1}$ Perinatal HIV Research Unit (PHRU), Faculty of Health Sciences, University of the Witwatersrand, Johannesburg, South Africa; ${ }^{2}$ Faculty of Health Sciences, Simon Fraser University, Burnaby, Canada; ${ }^{3}$ MatCH Research Unit (MRU), Department of Gynecology and Obstetrics, Faculty of Health Sciences, University of the Witwatersrand, Johannesburg, South Africa; ${ }^{4}$ South African Medical Research Council, Cape Town, South Africa

\subsection{6/sextrans-2019-sti.333}

Background South African young women and other key populations have amongst the highest HIV incidence globally, and antiretroviral treatment (ART) initiation remains challenging. We measured HIV incidence, ART initiation, adherence, status disclosure and related support within 3 months from HIV diagnosis for youth who acquired HIV during follow-up study visits.

Methods The AYAZAZI cohort study enrolled 425 selfreported HIV-negative or status-unknown 16-24 year olds from Soweto and Durban, South Africa (2014-2016). Participants completed interviewer-administered questionnaires (assessing socio-demographics, sexual behaviour and history, HIV-related service utilization, ART adherence, HIV disclosure and support) and underwent HIV rapid testing at enrolment and every 6 months for 4 visits.

Results 13/425(3.1\%) enrollees were HIV-infected at enrolment. 12/412(2.8\%; $\mathrm{n}=5$ Durban, $\mathrm{n}=7$ Soweto) with median age of 19 (IQR17-21) years, acquired HIV during their first year of follow-up $(\mathrm{IR}=2.54[95 \% \mathrm{CI}: 1.5-4.4]$ per 100 personyears); 6/12 were females, 5/12(42\%) identified as LGBTQ, $10 / 12(83 \%)$ were sexually active and 5/10(50\%) used a condom at last sex.Three months after HIV diagnosis, 10/12 (83\%) reported they were medically advised to initiate ART, and $8 / 12(67 \%)$ had initiated ART. Fear of provider judgement was the most common reason for ART non-initiation (3/ 4;75\%). A third (4/12) had not accessed any HIV-related medical or support services since diagnosis, commonly because of a disbelief of HIV test results $(4 / 4 ; 100 \%)$. Of those who initiated ART, 5/8(63\%) said they forgot a dose once or more per week. Eighty-three percent (10/12) had disclosed their HIV status to someone. The most common source of support related to HIV was from friends (7/12; 58\%). 2/12(17\%) rated that taking ART makes the risk of HIV infection a lot lower.

Conclusion HIV incidence was 2.5 per 100 person-years amongst our youth cohort; immediate ART uptake and adherence was sub-optimal; awareness of HIV prevention benefits of ART was low. Biomedical technologies and improved support systems for HIV-infected youth are needed.

Disclosure No significant relationships.

\section{P179 COMMUNITY BASED LOCAL RAPID HIV TESTING CAMPAIGNS TO EXPAND HIV DIAGNOSTIC COVERAGE}

${ }^{1}$ Ana Amélia Bones*, ${ }^{2}$ Mcarthur Barrow, ${ }^{3}$ Carlos Andres Casas, ${ }^{4}$ Marina Dias, ${ }^{4}$ Gabriela Storck, ${ }^{1}$ Silvio Cazella, ${ }^{5}$ Airton Stein. ${ }^{1}$ Health Federal University from Porto Alegre, Health Informatics Post Graduation Program, Porto Alegre, Brazil; ${ }^{2}$ Municipal Secretary of Health of Porto Alegre, Primary Health Care Trainee Program, St.Philip, Barbados; ${ }^{3}$ Health Federal University from Porto Alegre, Medicine Academic, Bogota, Colombia; ${ }^{4}$ Municipal Secretary of Health of Porto Alegre, Departamento of Sexually Transmitted Infections, Porto Alegre, Brazil; ${ }^{5}$ Health Federal University from Porto Alegre, Health Science Post Graduation Program, Porto Alegre, Brazil

10.1136/sextrans-2019-sti.334

Background The UNAIDS 90-90-90 programme aims to increase healthcare access for asymptomatic HIV patients, ensuring their well-being and disrupting the transmission network of the virus. The city of Porto Alegre, is one of the cities with the worst HIV indicators in Latin America. One of the strategies currently being implemented to address this is to develop a public Rapid Testing (RT) campaign, with immediate management of HIV, Syphilis and Hepatitis C. The objective of this research is to evaluate the initial results of the strategy in 2018.

Methods Cross-sectional study using the time series approach for all RTs conducted in public testing events in 2018, through data mining, with WEKA software.

Results Among the 4157 RTs carried out, reactive test results were observed as follows: 97(2.3\%) HIV, 626(15\%) syphilis and $156(3.7 \%)$ for Hepatitis C. The characteristics of this population are: 2004(48\%) women, 375(9\%) homo/bisexual, $710(17 \%)$ under 25 years and 1001(24\%) elderly. In the HIV reactive subgroup, 29(30\%) women, 29(30\%) homo/bisexual, 11(11\%) under 25 years, 17(17\%) elderly, 54(56\%) syphilis co-infection and $14(14 \%)$ for Hepatitis C reactive test, it should be noted that these results differ in proportion to published municipal/national data.

Conclusion The availability of RTs in public spaces facilitated early diagnosis in young and homosexual/bisexual populations. However, the campaign also observed participation from other populations such as the elderly and homeless, who are guaranteed by law priority access to health services. The amplification of healthcare access through RTs made early diagnosis possible in asymptomatic individuals. According to data mining, this strategy reached population groups which differ from those observed in Epidemiological Bulletins of previous years. The recommendation to also encourage the provision of ART to the elderly and homeless can be an effective strategy that should be evaluated in future research.

Disclosure No significant relationships.

\section{P180 VIRTUAL ACADEMIC DETAILING TO IMPROVE QUALITY OF HIV HEALTH CARE}

${ }^{1}$ Ana Amélia Bones*, ${ }^{2}$ Edilson Oliveira Junior, ${ }^{1}$ Silvio Cazella, ${ }^{3}$ Airton Stein. ${ }^{1}$ Health Federal University from Porto Alegre, Health Informatics Post Graduation Program, Porto Alegre, Brazil; ${ }^{2}$ Health Federal University from Porto Alegre, Biohealth Informatics Graduation, Porto Alegre, Brazil; ${ }^{3}$ Health Federal University from Porto Alegre, Health Science Post Graduation Program, Porto Alegre, Brazil

10.1136/sextrans-2019-sti.335

Background Academic Detailing (AD) is a scientific dissemination strategy that is characterized by the one-by-one orientation to improve the quality of care. It has been used 